Tropical Journal of Pharmaceutical Research May 2017; 16 (5): 1157-1165

ISSN: $1596-5996$ (print); 1596-9827 (electronic)

(c) Pharmacotherapy Group, Faculty of Pharmacy, University of Benin, Benin City, 300001 Nigeria.

All rights reserved.

Available online at http://www.tjpr.org

Original Research Article

http://dx.doi.org/10.4314/tjpr.v16i5.25

\title{
Synthesis and molecular docking of new hydrazones derived from ethyl isonipecotate and their biological activities
}

\author{
A Munir ${ }^{1}$, Aziz-ur-Rehman ${ }^{1 *}$, MA Abbasi ${ }^{1}$, SZ Siddiqui ${ }^{1}$, A Nasir ${ }^{2}$, SG Khan ${ }^{3}$, S \\ Rasool $^{1}$ and SAA Shah ${ }^{4,5}$ \\ ${ }^{1}$ Department of Chemistry, Government College University, Lahore-54000, ${ }^{2}$ Human Molecular Genetic Lab (HMG), Faculty of \\ Biological Science, Department of Biochemistry Quaid-I-Azam University Islamabad, ${ }^{3}$ Department of Chemistry, Government \\ College University, Faisalabad, Pakistan, ${ }^{4}$ Faculty of Pharmacy, Universiti Teknologi Mara, Puncak Alam Campus, 42300 \\ Bandar Puncak Alam, Selangor Darul Ehsan, Malaysia, ${ }^{5}$ Atta-ur-Rahman Institute for Natural Products Discovery (AuRIns), \\ Universiti Teknologi Mara, Puncak Alam Campus, 42300 Bandar Puncak Alam, Selangor Darul Ehsan, Malaysia
}

*For correspondence: Email: azizryk@yahoo.com, rehman@gcu.edu.pk; Tel: +92-42-111000010

Revised accepted: 16 April 2017

\begin{abstract}
Purpose: To investigate the antibacterial and $\alpha$-glucosidase inhibitory activities of hydrazone derivatives (8a-h) of ethyl isonipecotate.

Methods: The reaction of ethyl isonipecotate (2) with 3,5-dichloro-2-hydroxybenzenesulfonyl chloride (1) in an aqueous basic medium yielded ethyl 1-[(3,5-dichloro-2-hydroxyphenyl)sulfonyl]piperidin-4carboxylate (3). Compound $\mathbf{3}$ was subsequently converted to ethyl 1-[(3,5-dichloro-2ethoxyphenyl)sulfonyl]piperidin-4-carboxylate (5) via O-alkylation. Compound 5 on reaction with hydrated hydrazine yielded 1-[(3,5-dichloro-2-ethoxyphenyl)sulfonyl]piperidin-4-carbohyrazide (6) in $\mathrm{MeOH}$. Target compounds $\mathbf{8 a}-\boldsymbol{h}$ were synthesized by stirring $\mathbf{6}$ with different aromatic aldehydes (7a-h) in $\mathrm{MeOH}$. All the synthesized compounds were structurally elucidated by proton nuclear magnetic resonance ( $\left.{ }^{\prime} H-N M R\right)$, electron impact mass spectrometry (EI-MS) and infrared (IR) spectroscopy. For antibacterial activity, solutions of the synthesized compounds were mixed with bacterial strains, and the change in absorbance before and after incubation was determined. For enzyme inhibitory activity, change in the absorbance of mixtures of synthesized compounds and enzyme before and after incubation with substrate was determined.

Results: The target compounds were synthesized in appreciable yields and well characterized by spectral data analysis. Salmonella typhi was inhibited by $8 \boldsymbol{e}$ (MIC $8.00 \pm 0.54 \mu \mathrm{M}$ ), Escherichia coli by $8 f$ $(8.21 \pm 0.83 \mu \mathrm{M})$, Bacillus subtilis by $8 \mathrm{c}(8.56 \pm 0.63 \mu \mathrm{M})$ and Staphylococcus aureus by $8 \mathrm{c}(8.86 \pm 0.29$ $\mu M)$. Two compounds, $8 \mathbf{e}$ and $\mathbf{8 d}$, were very effective inhibitors of $\alpha$-glucosidase with $I_{50}$ values of $40.62 \pm 0.07$ and $48.64 \pm 0.08 \mu \mathrm{M}$, respectively.

Conclusion: Low $I C_{50}$ values of the synthesized compounds against $\alpha$-glucosidase demonstrates their potential in type-2 diabetes treatment. Furthermore, these compounds exhibit substantial antibacterial activity against the bacterial strains tested.
\end{abstract}

Keywords: Antibacterial activity, a-Glucosidase inhibition, Ethyl isonipecotate, Hydrazones

Tropical Journal of Pharmaceutical Research is indexed by Science Citation Index (SciSearch), Scopus, International Pharmaceutical Abstract, Chemical Abstracts, Embase, Index Copernicus, EBSCO, African Index Medicus, JournalSeek, Journal Citation Reports/Science Edition, Directory of Open Access Journals (DOAJ), African Journal Online, Bioline International, Open-J-Gate and Pharmacy Abstracts

\section{INTRODUCTION}

The labile lone pair of nitrogens in hydrazones is known to play an active role in the bioactivity and chemical reactivity of this class of compounds $[1,2]$. These compounds have been shown to have anti-tuberculosis [3], antimicrobial [4], antimycotic [5] and antihypertensive activities [6]. 
Furthermore, industrial chemistry has found many applications for hydrazones [7-9]. $\alpha$ Glucosidase (EC 3.2.1.20) is an enzyme that acts on 1,4-alpha bonds [10,11].

The cleavage of carbohydrates is delayed by $\alpha$ glucosidase inhibitors (AGI) in the small intestine $[12,13]$. In search of bioactive compounds, an attempt was made to synthesize some new hydrazones bearing a sulfamoyl moiety.

\section{EXPERIMENTAL}

All chemical reagents were from Merck, Sigma Aldrich and Alfa Aesar and purchased through local suppliers, and they were used without further purification. Purity of the synthesized compounds was assured by thin layer chromatography (TLC); the plates were developed with $n$-hexane and ethyl acetate solvent systems and visualized under UV at 254 $\mathrm{nm}$ and also by spraying with ceric sulfate solution.

The melting points of all synthesized compounds were determined using open capillary tubes on a Griffin and George apparatus. IR spectra were recorded using the potassium bromide pellet method on a Jasco-320-A spectrophotometer with wave number in $\mathrm{cm}^{-1} .{ }^{1} \mathrm{H}-\mathrm{NMR}$ spectra were recorded in $\mathrm{CDCl}_{3}$ on a Bruker spectrometer operating at $400 \mathrm{MHz}$. Chemical shift values are reported in ppm $(\delta)$ units taking TMS as reference and the coupling constants $(J)$ are in $\mathrm{Hz}$. Mass spectra (EIMS) were recorded on a JMS-HX-110 spectrometer.

\section{Procedure for synthesis of sulfonamide (3) in aqueous medium}

Ethyl isonipecotate $(6.49 \mathrm{mmol} ; 1 \mathrm{~mL}, 2)$ was added to a $100-\mathrm{mL}$ round-bottom flask containing $15 \mathrm{~mL}$ basic aqueous medium prepared by addition of aqueous $\mathrm{Na}_{2} \mathrm{CO}_{3}$ with $\mathrm{pH}$ adjusted to 8-9. Next, 3,5-dichloro-2-hydroxybenzenesulfonylchloride $(6.49 \mathrm{mmol} ; 1.698 \mathrm{~g}, 1)$ was gradually added to the reaction flask over 5-10 min. The reaction mixture was stirred for 6-8 $\mathrm{h}$ with $\mathrm{pH}$ maintained at 8-9 at ambient temperature. The completion of the reaction was checked by TLC until confirmed by a single spot. Product $\mathbf{3}$ was collected by acidifying the reaction mixture with dilute $\mathrm{HCl}$ to bring the $\mathrm{pH}$ to $5-7$. The synthesized product was collected by filtration, washed with distilled water and dried for next use.

\section{Procedure for synthesis of 0 -alkyl derivative (5)}

Compound 3 (0.5249 mmol, $0.2 \mathrm{~g}$ ) was dissolved in DMF $(5 \mathrm{~mL})$ in a $100-\mathrm{mL}$ round-bottom flask. Solid $\mathrm{KOH}(0.5249 \mathrm{mmol}, 0.0293 \mathrm{~g})$ was added to activate the $\mathrm{O}$-substitution. Ethyl iodide $(0.5249 \mathrm{mmol} ; 42.2 \mu \mathrm{L}, 4)$ was then added, and the mixture was stirred for $2-3 \mathrm{~h}$ at $60{ }^{\circ} \mathrm{C}$. Completion of the reaction was confirmed by TLC showing a single spot. Chilled distilled water was used to collect the precipitates of pure product $\mathbf{5}$. Precipitates were filtered, washed with distilled water and dried.

\section{Procedure for synthesis of carbohydrazide (6)}

Compound 5 (1.467 mmol, $0.6 \mathrm{~g}$ ) was dissolved in $\mathrm{MeOH}(5-10 \mathrm{~mL})$ in a $100-\mathrm{mL}$ round-bottom flask and stirred for 2-5 min. Hydrazine hydrate $(1.467 \mathrm{mmol})$ was then added and the reaction mixture was refluxed for $6 \mathrm{~h}$. There was a color change in the reaction mixture from orangeyellow to grey as the reaction proceeded toward completion. The progress of the reaction was followed by TLC. After reaction completion, the mixture was quenched with cold distilled water and the precipitates of 6 were filtered, washed with distilled water and dried for further use.

\section{General procedure for synthesis of various hydrazone derivatives (8a-h)}

A methanolic solution of compound 6 (0.2523 $\mathrm{mmol}, 0.1 \mathrm{~g}$ ) was placed in a $50-\mathrm{mL}$ roundbottom flask and stirred at room temperature. Aromatic aldehydes (7a-h) were introduced in equimolar ratios to produce hydrazone derivatives. The reaction duration for different aldehydes varied 2-3 h. Reaction completion was confirmed by TLC, and distilled water was added to the mixture to precipitate the compounds synthesized, 8a-h, which were filtered, washed with distilled water and dried.

\section{Evaluation of antibacterial activity}

The broth microdilution method was employed to test for antibacterial activity [14]. The solutions of synthesized compounds were mixed with bacterial strains and change in absorbance before and after incubation was determined.

\section{$\alpha$-Glucosidase assay}

$\alpha$-Glucosidase inhibitory activity was determined as previously described, with slight modification [15]. 
<smiles>CCOC(OCC)C1CCNCC1</smiles><smiles>CCOC(=O)C1CCN(S(=O)(=O)c2cc(Cl)cc(Cl)c2O)CC1</smiles>

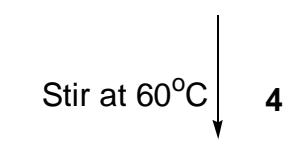<smiles>CCOc1c(Cl)cc(Cl)cc1S(=O)(=O)N1CCC(C(=O)NN)CC1</smiles><smiles>CC(C)C(C)CO</smiles><smiles>CCOC(=O)C1CCN(S(=O)(=O)c2cc(Cl)cc(Cl)c2OCC)CC1</smiles><smiles>[R]CC(C)C(Br)CC</smiles><smiles>[R]C=NNC(=O)C1CCN(S(=O)(=O)c2cc(Cl)cc(Cl)c2OC[CH2])CC1</smiles>

Compd. Compd.

Scheme 1: Synthetic scheme for hydrazone derivatives of 1-[(3,5-dichloro-2-ethoxyphenyl)sulfonyl]piperdine-4carbohydrazide

Enzyme activity was based on the difference in absorbance of the mixture of synthesized compounds and enzyme before and after incubation with substrate.

\section{Molecular docking study}

The crystallographic structure of Saccharomyces cerevisiae isomaltase (PDB code 3AJ7; resolution $1.30 \AA$ ) showing $72.4 \%$ sequence identity with the target was selected as a template. The 3D structure of a-glucosidase for Saccharomyces cerevisiae was predicted using the Molecular Operating Environment (MOE 2010.11) software package. MOE docking program was used to analyze the binding modes of the ligands with the protein molecule. The best conformations were analyzed for hydrogen bonding and $\pi-\pi$ interactions [16-18].

\section{Statistical analysis}

The results are presented as mean \pm SEM $(n=$ 3 ) and were analyzed by Microsoft Excel 2010. The results for $50 \%$ inhibitory concentration $\left(\mathrm{IC}_{50}\right)$ and minimum inhibitory concentration (MIC) were obtained at different dilutions (5 - 30 $\mu \mathrm{g} / \mathrm{well}$ ) and analyzed by EZ-Fit software (Perrella Scientific Inc., Amherst, USA).

\section{RESULTS}

1-(3,5-Dichloro-2-ethoxybenzenesulfonyl)- $N$ '(arylidene)piperidin-4-carbohydrazides, $\mathbf{8 a - h}$, were prepared according to the protocol 
described in Scheme 1. The synthesized compounds exhibited substantial $\alpha$-glucosidase inhibitory activity and antibacterial activity.

Ethyl 1-[(3,5-dichloro-2-hydroxyphenyl)sulfonyl]piperidin-4-carboxylate (3)

White powder; Yield: $87 \%$; IR $(\mathrm{KBr})$ : $\mathrm{u}_{\max }$ : 3247 (O-H), $3110(\mathrm{Ar}-\mathrm{H}), 2860(\mathrm{C}-\mathrm{H}), 1750(>\mathrm{C}=\mathrm{O})$, 1610 (Aromatic $\mathrm{C}=\mathrm{C}), 1386\left(-\mathrm{SO}_{2}\right) ;{ }^{1} \mathrm{H}-\mathrm{NMR}(400$ $\left.\mathrm{MHz}, \mathrm{CDCl}_{3}\right): \delta 8.92\left(\mathrm{~s}, 1 \mathrm{H}, \mathrm{HO}-2^{\prime}\right), 7.77(\mathrm{~d}, J=$ $\left.2.8 \mathrm{~Hz}, 1 \mathrm{H}, \mathrm{H}-6^{\prime}\right), 7.55$ (d, J = 2.4 Hz, 1H, H-4'), 4.12 ( $\mathrm{q}, J=6.8 \mathrm{~Hz}, 2 \mathrm{H}, \mathrm{H}-7), 3.80-3.77(\mathrm{~m}, 2 \mathrm{H}$, $\mathrm{H}_{e}-2$ \& $\left.\mathrm{H}_{e}-6\right), 3.34-3.04(\mathrm{~m}, 1 \mathrm{H}, \mathrm{H}-4), 2.88-2.83$ (m, 2H, $\mathrm{H}_{\mathrm{a}}-2$ \& $\left.\mathrm{H}_{\mathrm{a}}-6\right)$, 2.05-1.96 (m, 2H, $\mathrm{H}_{\mathrm{e}}-3$ \& $\left.\mathrm{H}_{e}-5\right), 1.86-1.77\left(\mathrm{~m}, 2 \mathrm{H}, \mathrm{H}_{\mathrm{a}}-3 \& \mathrm{H}_{\mathrm{a}}-5\right), 1.47(\mathrm{t}, J=$ $6.8 \mathrm{~Hz}, 3 \mathrm{H}, \mathrm{H}-8)$; EIMS: $\mathrm{m} / \mathrm{z} 381 \mathrm{[M}^{+}, 352$ $\left[\mathrm{C}_{12} \mathrm{H}_{12} \mathrm{Cl}_{2} \mathrm{NO}_{5} \mathrm{~S}\right]^{+}, \quad 336 \quad\left[\mathrm{C}_{12} \mathrm{H}_{12} \mathrm{Cl}_{2} \mathrm{NO}_{4} \mathrm{~S}\right]^{+}, \quad 308$ $\left[\mathrm{C}_{11} \mathrm{H}_{12} \mathrm{Cl}_{2} \mathrm{NO}_{3} \mathrm{~S}\right]^{+}, \quad 224\left[\mathrm{C}_{7} \mathrm{H}_{6} \mathrm{Cl}_{2} \mathrm{O}_{3} \mathrm{~S}\right]^{+}, \quad 156$ $\left[\mathrm{C}_{8} \mathrm{H}_{14} \mathrm{NO}_{2}\right]^{++}, 144\left[\mathrm{C}_{6} \mathrm{H}_{2} \mathrm{Cl}_{2}\right]^{++}, 111\left[\mathrm{C}_{6} \mathrm{H}_{9} \mathrm{NO}\right]^{++}, 83$ $\left[\mathrm{C}_{5} \mathrm{H}_{9} \mathrm{~N}\right]^{+}$.

\section{Ethyl 1-[(3,5-dichloro-2-ethoxyphenyl)sulfo- nyl]piperidin-4-carboxylate (5)}

White powder; Yield: $80 \%$; IR (KBr): U $U_{\max }: 3045$ (Ar-H), $2975(\mathrm{C}-\mathrm{H}), 1617$ (Aromatic $\mathrm{C}=\mathrm{C}$ ), 1369 $\left(-\mathrm{SO}_{2}\right), 1170$ (C-O-C); ${ }^{1} \mathrm{H}-\mathrm{NMR} \quad(400 \mathrm{MHz}$, $\mathrm{CDCl}_{3}$ ): $\delta 7.79\left(\mathrm{~d}, J=2.8 \mathrm{~Hz}, 1 \mathrm{H}, \mathrm{H}-6^{\prime}\right), 7.53(\mathrm{~d}$, $\left.J=2.4 \mathrm{~Hz}, 1 \mathrm{H}, \mathrm{H}-4^{\prime}\right), 4.22(\mathrm{q}, J=6.8 \mathrm{~Hz}, 2 \mathrm{H}, \mathrm{H}-$ 7'), 3.88 (q, $J=6.4 \mathrm{~Hz}, 2 \mathrm{H}, \mathrm{H}-7), 3.82-3.57$ (m, $2 \mathrm{H}, \mathrm{H}_{e}-2$ \& $\left.\mathrm{H}_{e}-6\right), 2.32-2.04(\mathrm{~m}, 1 \mathrm{H}, \mathrm{H}-4), 2.77-$ $2.73\left(\mathrm{~m}, 2 \mathrm{H}, \mathrm{H}_{a^{-}}-2\right.$ \& $\left.\mathrm{H}_{\mathrm{a}}-6\right)$, 2.10-1.96 (m, 2H, $\mathrm{H}_{e^{-}}$ $\left.3 \& \mathrm{H}_{e}-5\right), 1.80-1.72\left(\mathrm{~m}, 2 \mathrm{H}, \mathrm{H}_{\mathrm{a}}-3 \& \mathrm{H}_{a}-5\right), 1.42$ (t, $\left.J=6.8 \mathrm{~Hz}, 3 \mathrm{H}, \mathrm{H}-8^{\prime}\right), 1.31$ (t, $J=6.4 \mathrm{~Hz}, 3 \mathrm{H}$, $\mathrm{H}-8$ ); EIMS: $m / z 409[\mathrm{M}]^{+}, 380\left[\mathrm{C}_{14} \mathrm{H}_{16} \mathrm{Cl}_{2} \mathrm{NO}_{5} \mathrm{~S}\right]^{+}$, $364 \quad\left[\mathrm{C}_{14} \mathrm{H}_{16} \mathrm{Cl}_{2} \mathrm{NO}_{4} \mathrm{~S}\right]^{+}$, $336 \quad\left[\mathrm{C}_{13} \mathrm{H}_{16} \mathrm{Cl}_{2} \mathrm{NO}_{3} \mathrm{~S}\right]^{+}$, $188 \quad\left[\mathrm{C}_{8} \mathrm{H}_{7} \mathrm{Cl}_{2} \mathrm{O}\right]^{++}, \quad 156 \quad\left[\mathrm{C}_{8} \mathrm{H}_{14} \mathrm{NO}_{2}\right]^{+}, \quad 144$ $\left[\mathrm{C}_{6} \mathrm{H}_{2} \mathrm{Cl}_{2}\right]^{++}, 111\left[\mathrm{C}_{6} \mathrm{H}_{9} \mathrm{NO}\right]^{+}, 83\left[\mathrm{C}_{5} \mathrm{H}_{9} \mathrm{~N}\right]^{++}$.

\section{1-(3,5-Dichloro-2-ethoxyphenylsulfonyl) piperidin-4-carbohydrazide (6)}

Grey powder; Yield: 79 \%; IR (KBr): $U_{\max }: 3332$ (CON-H), 3029 (Ar-H), 1639 (>C=O), 1610 (Aromatic C=C), $1378\left(-\mathrm{SO}_{2}\right), 1159(\mathrm{C}-\mathrm{O}-\mathrm{C}) ;{ }^{1} \mathrm{H}-$ NMR $\left(400 \mathrm{MHz}, \mathrm{CDCl}_{3}\right): \delta 8.37$ (br s, CON-H), $7.77\left(\mathrm{~d}, J=2.8 \mathrm{~Hz}, 1 \mathrm{H}, \mathrm{H}-6^{\prime}\right), 7.56(\mathrm{~d}, J=2.4$ $\mathrm{Hz}, 1 \mathrm{H}, \mathrm{H}-4^{\prime}$ ), 3.88 (q, J = $6.4 \mathrm{~Hz}, 2 \mathrm{H}, \mathrm{H}-7^{\prime}$ ), 3.81-3.77 (m, 2H, $\mathrm{H}_{e}-2$ \& $\left.\mathrm{H}_{e}-6\right)$, 2.88-2.83 (m, $1 \mathrm{H}, \mathrm{H}-4), 2.67-2.53\left(\mathrm{~m}, 2 \mathrm{H}, \mathrm{H}_{\mathrm{a}}-2\right.$ \& $\left.\mathrm{H}_{\mathrm{a}}-6\right)$, 2.05$1.96\left(\mathrm{~m}, 2 \mathrm{H}, \mathrm{H}_{e}-3 \& \mathrm{H}_{e^{-}}\right.$), 1.86-1.77 (m, 2H, $\mathrm{H}_{a^{-}}$ $\left.3 \& \mathrm{H}_{a}-5\right), 1.47$ (t, $\left.J=7.2 \mathrm{~Hz}, 3 \mathrm{H}, \mathrm{H}-8^{\prime}\right)$; EIMS: $\mathrm{m} / \mathrm{z} 395 \quad[\mathrm{M}]^{++}, \quad 365 \quad\left[\mathrm{C}_{14} \mathrm{H}_{17} \mathrm{Cl}_{2} \mathrm{NO}_{4} \mathrm{~S}\right]^{*+}, \quad 364$ $\left[\mathrm{C}_{14} \mathrm{H}_{16} \mathrm{Cl}_{2} \mathrm{NO}_{4} \mathrm{~S}\right]^{+}, \quad 336 \quad\left[\mathrm{C}_{13} \mathrm{H}_{16} \mathrm{Cl}_{2} \mathrm{NO}_{3} \mathrm{~S}\right]^{+}, 142$ $\left[\mathrm{C}_{6} \mathrm{H}_{12} \mathrm{~N}_{3} \mathrm{O}\right]^{+}, 111\left[\mathrm{C}_{6} \mathrm{H}_{9} \mathrm{NO}\right]^{+}, 83\left[\mathrm{C}_{5} \mathrm{H}_{9} \mathrm{~N}\right]^{+}$.
1-(3,5-Dichloro-2-ethoxyphenylsulfonyl)- $N$-(4methoxybenzylidene)piperidin-4-carbohydrazide (8a)

White powder; Yield: $96 \%$; IR (KBr): $U_{\max }: 3025$ (Ar-H stretching), $2869(\mathrm{C}-\mathrm{H}), 1678(\mathrm{C}=\mathrm{N}), 1508$ (Aromatic C=C), $1360\left(-\mathrm{SO}_{2}\right), 1162(\mathrm{C}-\mathrm{O}-\mathrm{C}) ;{ }^{1} \mathrm{H}-$ $\operatorname{NMR}\left(400 \mathrm{MHz}, \mathrm{CDCl}_{3}\right): \delta 8.47$ (br s, $1 \mathrm{H}, \mathrm{CONH}$ ), $8.38(\mathrm{~s}, 1 \mathrm{H}, \mathrm{H}-7 "), 7.80(\mathrm{~d}, J=2.7 \mathrm{~Hz}, 1 \mathrm{H}, \mathrm{H}-$ 6'), $7.58\left(\mathrm{~d}, J=2.5 \mathrm{~Hz}, 1 \mathrm{H}, \mathrm{H}-4^{\prime}\right), 7.71(\mathrm{~d}, J=8.2$ $\mathrm{Hz}, 2 \mathrm{H}, \mathrm{H}-2$ " \& H-6"), 7.21 (d, $J=8.4 \mathrm{~Hz}, 2 \mathrm{H}, \mathrm{H}-$ 3" \& H-5"), 4.23 (q, J = 6.4 Hz, H-7'), 3.81 (s, 3H, $\left.\mathrm{OCH}_{3}-8 "\right)$, 3.89-3.84 (m, $2 \mathrm{H}, \mathrm{H}_{e}-2$ \& $\left.\mathrm{H}_{e}-6\right)$, 3.36$3.20(\mathrm{~m}, 1 \mathrm{H}, \mathrm{H}-4), 2.86-2.80\left(\mathrm{~m}, 2 \mathrm{H}, \mathrm{H}_{\mathrm{a}}-2\right.$ \& $\mathrm{H}_{a^{-}}$ 6), 1.96-1.80 (m, 4H, H-3 \& H-5), 1.43 (t, $J=6.4$ $\left.\mathrm{Hz}, 3 \mathrm{H}, \quad \mathrm{H}-8^{\prime}\right)$; EIMS: $\mathrm{m} / \mathrm{z} 513\left[\mathrm{M}^{\circ+}, 379\right.$ $\left[\mathrm{C}_{14} \mathrm{H}_{17} \mathrm{Cl}_{2} \mathrm{~N}_{2} \mathrm{O}_{4} \mathrm{~S}\right]^{+}, 364\left[\mathrm{C}_{14} \mathrm{H}_{16} \mathrm{Cl}_{2} \mathrm{NO}_{4} \mathrm{~S}\right]^{+}, 336$ $\left[\begin{array}{llll}\left.\mathrm{C}_{13} \mathrm{H}_{16} \mathrm{Cl}_{2} \mathrm{NO}_{3} \mathrm{~S}\right]^{+}, & 260 & {\left[\mathrm{C}_{14} \mathrm{H}_{18} \mathrm{~N}_{3} \mathrm{O}_{2}\right]^{++},} & 147\end{array}\right.$ $\left[\mathrm{C}_{5} \mathrm{H}_{9} \mathrm{NO}_{2} \mathrm{~S}\right]^{+}, 134\left[\mathrm{C}_{8} \mathrm{H}_{8} \mathrm{NO}_{2}\right]^{++}, 107\left[\mathrm{C}_{7} \mathrm{H}_{7} \mathrm{O}\right]^{+}$, $83\left[\mathrm{C}_{5} \mathrm{H}_{9} \mathrm{~N}\right]^{*+}$.

1-(3,5-Dichloro-2-ethoxyphenylsulfonyl)- $N$-(4hydroxybenzylidene)piperidin-4-carbohydrazide (8b)

Off-white powder; Yield: $96 \%$; IR (KBr): $\mathrm{U}_{\max }$ : $3135($ Ar-H), $2892(\mathrm{C}-\mathrm{H}), 1680(\mathrm{C}=\mathrm{N}), 1588$ (Aromatic $\mathrm{C}=\mathrm{C}), 1395\left(-\mathrm{SO}_{2}\right), 1134(\mathrm{C}-\mathrm{O}-\mathrm{C}) ;{ }^{1} \mathrm{H}-$ NMR $\left(400 \mathrm{MHz}, \mathrm{CDCl}_{3}\right): \delta 9.95(\mathrm{br} \mathrm{s}, 1 \mathrm{H}, \mathrm{CONH}$ ), 9.93 (s, 1H, OH-8"), 8.29 (s, 1H, H-7"), 7.90 (d, $\left.J=2.8 \mathrm{~Hz}, 1 \mathrm{H}, \mathrm{H}-6^{\prime}\right), 7.83(\mathrm{~d}, J=2.4 \mathrm{~Hz}, 1 \mathrm{H}, \mathrm{H}-$ 4'), 7.51 (d, $J=8.8 \mathrm{~Hz}, 2 \mathrm{H}, \mathrm{H}-2$ " \& H-6"), 6.80 (d, $J=8.4 \mathrm{~Hz}, 2 \mathrm{H}, \mathrm{H}-3$ " \& H-5"), $4.23(\mathrm{q}, J=6.5 \mathrm{~Hz}$, $\left.2 \mathrm{H}, \mathrm{H}-\mathrm{7}^{\prime}\right), 3.68-3.56\left(\mathrm{~m}, 2 \mathrm{H}, \mathrm{H}_{e}-2\right.$ \& $\left.\mathrm{H}_{e}-6\right)$, 3.42$3.20(\mathrm{~m}, 1 \mathrm{H}, \mathrm{H}-4), 2.86-2.82(\mathrm{dt}, J=11.2 \mathrm{~Hz}$, $2 \mathrm{H}, \mathrm{H}_{\mathrm{a}}-2$ \& $\mathrm{H}_{\mathrm{a}}-6$ ), 1.95-1.84 (m, 4H, $\mathrm{H}_{e}-3$ \& $\mathrm{H}_{e}-5$, $\mathrm{H}_{\mathrm{a}}-3 \& \mathrm{H}_{\mathrm{a}}-5$ ), 1.43 (t, $\left.J=3.2 \mathrm{~Hz}, 3 \mathrm{H}, \mathrm{H}-8^{\prime}\right)$; EIMS: $\mathrm{m} / \mathrm{z} \quad 499 \quad[\mathrm{M}]^{+}, \quad 379 \quad\left[\mathrm{C}_{14} \mathrm{H}_{17} \mathrm{Cl}_{2} \mathrm{~N}_{2} \mathrm{O}_{4} \mathrm{~S}\right]^{+}, \quad 336$

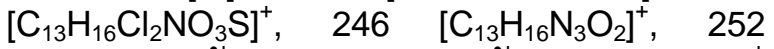
$\left[\mathrm{C}_{8} \mathrm{H}_{7} \mathrm{Cl}_{2} \mathrm{O}_{3} \mathrm{~S}\right]^{++}, 163\left[\mathrm{C}_{8} \mathrm{H}_{7} \mathrm{~N}_{2} \mathrm{O}_{2}\right]^{+}, 120\left[\mathrm{C}_{7} \mathrm{H}_{6} \mathrm{NO}^{+}\right.$ , $118\left[\mathrm{C}_{8} \mathrm{H}_{8} \mathrm{~N}\right]^{++}, 83\left[\mathrm{C}_{5} \mathrm{H}_{9} \mathrm{~N}\right]^{+}$.

1-(3,5-Dichloro-2-ethoxyphenylsulfonyl)- $N$-(3nitrobenzylidene)piperidin-4-carbohydrazide (8c)

White powder; Yield: $96 \%$; IR (KBr): $\mathrm{U}_{\max }: 3015$ (Ar-H), $2910(\mathrm{C}-\mathrm{H}), 1645(\mathrm{C}=\mathrm{N}), 1598$ (Aromatic $\mathrm{C}=\mathrm{C}), 1406\left(-\mathrm{SO}_{2}\right), 1122(\mathrm{C}-\mathrm{O}-\mathrm{C}) ;{ }^{1} \mathrm{H}-\mathrm{NMR}(400$ $\mathrm{MHz}, \mathrm{CDCl}_{3}$ ): $\delta 8.71$ (s, $1 \mathrm{H}, \mathrm{H}-2$ "), 8.32 (dd, $J=$ 8.4, $1.6 \mathrm{~Hz}, 1 \mathrm{H}, \mathrm{H}-4 "), 8.30$ (s, $\left.1 \mathrm{H}, \mathrm{H}-7^{\prime \prime}\right), 7.94$ (d, $J=7.6 \mathrm{~Hz}, 1 \mathrm{H}, \mathrm{H}-6 "), 7.80(\mathrm{~d}, J=2.8 \mathrm{~Hz}, 1 \mathrm{H}, \mathrm{H}-$ 6'), 7.72 (t, $J=8.0 \mathrm{~Hz}, 1 \mathrm{H}, \mathrm{H}-5 "), 7.58(\mathrm{~d}, J=$ $\left.2.4 \mathrm{~Hz}, 1 \mathrm{H}, \mathrm{H}-4^{\prime}\right), 4.24\left(\mathrm{q}, J=6.4 \mathrm{~Hz}, 2 \mathrm{H}, \mathrm{H}-7^{\prime}\right)$, 3.69-3.56 $\left(\mathrm{m}, \mathrm{H}_{e}-2\right.$ \& $\left.\mathrm{H}_{e}-6\right), 3.40-3.20(\mathrm{~m}, 1 \mathrm{H}, \mathrm{H}-$ 4), 2.90-2.86 (m, 2H, $\mathrm{H}_{a}-2$ \& $\left.\mathrm{H}_{\mathrm{a}}-6\right)$ ), 1.96-1.88 (m, $4 \mathrm{H}, \mathrm{H}-3$ \& $\mathrm{H}-5), 1.46\left(\mathrm{t}, \mathrm{J}=6.4 \mathrm{~Hz}, 3 \mathrm{H}, \mathrm{H}-8^{\prime}\right)$; EIMS: $m / z 528[\mathrm{M}]^{+}, 379\left[\mathrm{C}_{14} \mathrm{H}_{17} \mathrm{Cl}_{2} \mathrm{~N}_{2} \mathrm{O}_{4} \mathrm{~S}\right]^{+}, 364$ $\left[\mathrm{C}_{14} \mathrm{H}_{17} \mathrm{Cl}_{2} \mathrm{NO}_{4} \mathrm{~S}\right]^{+}, \quad 336\left[\mathrm{C}_{13} \mathrm{H}_{16} \mathrm{Cl}_{2} \mathrm{NO}_{3} \mathrm{~S}\right]^{+}, 275$ 
$\left[\mathrm{C}_{13} \mathrm{H}_{15} \mathrm{~N}_{4} \mathrm{O}_{3}\right]^{++}, 149\left[\mathrm{C}_{7} \mathrm{H}_{5} \mathrm{~N}_{2} \mathrm{O}_{2}\right]^{*+}, 126\left[\mathrm{C}_{6} \mathrm{H}_{10} \mathrm{~N}_{2} \mathrm{O}\right]^{+}$ $83\left[\mathrm{C}_{5} \mathrm{H}_{9} \mathrm{~N}\right]^{+}$.

\section{1-(3,5-Dichloro-2-ethoxyphenylsulfonyl)- $N$ - (2,4-dichlorobenzylidene)piperidin-4- carbohydrazide (8d)}

White powder; Yield: $96 \%$; IR (KBr): U $U_{\text {max }}: 3035$ $(\mathrm{Ar}-\mathrm{H}), 2967(\mathrm{C}-\mathrm{H}), 1635(\mathrm{C}=\mathrm{N}), 1595$ (Aromatic $\mathrm{C}=\mathrm{C}), 1425\left(-\mathrm{SO}_{2}\right), 1112(\mathrm{C}-\mathrm{O}-\mathrm{C}) ;{ }^{1} \mathrm{H}-\mathrm{NMR}(400$ $\mathrm{MHz}, \mathrm{CDCl}_{3}$ ): $\delta 8.41\left(\mathrm{~s}, 1 \mathrm{H}, \mathrm{H}-7 \mathrm{C}^{\prime}\right), 7.80$ (d, $J=$ $\left.2.8 \mathrm{~Hz}, 1 \mathrm{H}, \mathrm{H}-6^{\prime}\right), 7.58\left(\mathrm{~d}, J=8.0 \mathrm{~Hz}, 1 \mathrm{H}, \mathrm{H}-6^{\prime \prime}\right)$, 7.57 (dd, $J=8.0,2.0 \mathrm{~Hz}, 1 \mathrm{H}, \mathrm{H}-5$ "), $7.55(\mathrm{~d}, J=$ $\left.2.4 \mathrm{~Hz}, 1 \mathrm{H}, \mathrm{H}-4^{\prime}\right), 7.32$ (d, J = 2.0 Hz, 1H, H-3"), 4.23 (q, $\left.J=6.4 \mathrm{~Hz}, 2 \mathrm{H}, \mathrm{H}-7^{\prime}\right), 3.69-3.56(\mathrm{~m}, 2 \mathrm{H}$, $\mathrm{H}_{e}-2$ \& $\left.\mathrm{H}_{e}-6\right), 3.40-3.20(\mathrm{~m}, 1 \mathrm{H}, \mathrm{H}-4), 2.86-2.80$ (m, $2 \mathrm{H}, \mathrm{H}_{\mathrm{a}}-2$ \& $\left.\mathrm{H}_{\mathrm{a}}-6\right)$ ), 1.96-1.84 (m, 4H, H-3 \& H5), 1.43 (t, $J=6.8 \mathrm{~Hz}, 3 \mathrm{H}, \mathrm{H}-8$ '); EIMS: $m / z 551$ $[\mathrm{M}]^{++}, \quad 379 \quad\left[\mathrm{C}_{14} \mathrm{H}_{17} \mathrm{Cl}_{2} \mathrm{~N}_{2} \mathrm{O}_{4} \mathrm{~S}\right]^{++}, \quad 364$ $\left[\mathrm{C}_{14} \mathrm{H}_{17} \mathrm{Cl}_{2} \mathrm{NO}_{4} \mathrm{~S}\right]^{+}, \quad 336\left[\mathrm{C}_{13} \mathrm{H}_{16} \mathrm{Cl}_{2} \mathrm{NO}_{3} \mathrm{~S}\right]^{+}, 298$ $\left[\mathrm{C}_{13} \mathrm{H}_{14} \mathrm{Cl}_{2} \mathrm{~N}_{3} \mathrm{O}\right]{ }^{+}, 172\left[\mathrm{C}_{7} \mathrm{H}_{4} \mathrm{Cl}_{2} \mathrm{O}\right]^{+}, 145$ $\left[\mathrm{C}_{6} \mathrm{H}_{3} \mathrm{Cl}_{2}\right]^{+}, 126\left[\mathrm{C}_{6} \mathrm{H}_{10} \mathrm{~N}_{2} \mathrm{O}\right]^{+}, 83\left[\mathrm{C}_{5} \mathrm{H}_{9} \mathrm{~N}\right]^{+}$.

\section{1-(3,5-Dichloro-2-ethoxyphenylsulfonyl)- $N$-(2- hydroxybenzylidene)piperidin-4- carbohydrazide (8e)}

Off white powder; Yield: $99 \%$; IR (KBr): $U_{\max }$ : $3135(\mathrm{Ar}-\mathrm{H}), 2892(\mathrm{C}-\mathrm{H}), 1680(\mathrm{C}=\mathrm{N}), 1588$ (Aromatic $\mathrm{C}=\mathrm{C}), 1395\left(-\mathrm{SO}_{2}\right), 1134(\mathrm{C}-\mathrm{O}-\mathrm{C}) ;{ }^{1} \mathrm{H}-$ NMR $\left(400 \mathrm{MHz}, \mathrm{CDCl}_{3}\right): \delta 8.59(\mathrm{~s}, 1 \mathrm{H}, \mathrm{OH}-8 ")$, 8.54 (s, 1H, H-7"), 7.90 (d, J = 2.8 Hz, $\left.1 \mathrm{H}, \mathrm{H}-6^{\prime}\right)$, $7.81(\mathrm{~d}, J=7.6 \mathrm{~Hz}, 1 \mathrm{H}, \mathrm{H}-6 "), 7.56(\mathrm{~d}, J=2.4$ $\mathrm{Hz}, 1 \mathrm{H}, \mathrm{H}-4$ ), 7.53 (dd, J = 7.6, $1.6 \mathrm{~Hz}, 1 \mathrm{H}, \mathrm{H}-$ 3"), 7.23 (dt, J = 7.6, $2.0 \mathrm{~Hz}, 1 \mathrm{H}, \mathrm{H}-4 "), 6.90(\mathrm{dt}$, $J=7.6,2.0 \mathrm{~Hz}, 1 \mathrm{H}, \mathrm{H}-5 "), 4.22$ (q, $J=6.8 \mathrm{~Hz}$, $\left.2 \mathrm{H}, \mathrm{H}-7^{\prime}\right)$, 3.69-3.56 (m, 2H, $\left.\mathrm{H}_{e}-2 \& \mathrm{H}_{e}-6\right)$, 3.40$3.22(\mathrm{~m}, 1 \mathrm{H}, \mathrm{H}-4), 2.86-2.78\left(\mathrm{~m}, 2 \mathrm{H}, \mathrm{H}_{a^{-}}-2\right.$ \& $\mathrm{H}_{a^{-}}$ 6), 1.96-1.74 (m, 4H, H-3 \& H-5), $1.43(\mathrm{t}, J=6.4$ $\left.\mathrm{Hz}, \quad 3 \mathrm{H}, \quad \mathrm{H}-8^{\prime}\right)$; EIMS: $m / z \quad 499\left[\mathrm{M}^{+}, 379\right.$ $\left[\mathrm{C}_{14} \mathrm{H}_{17} \mathrm{Cl}_{2} \mathrm{~N}_{2} \mathrm{O}_{4} \mathrm{~S}\right]^{++}, 364\left[\mathrm{C}_{14} \mathrm{H}_{17} \mathrm{Cl}_{2} \mathrm{NO}_{4} \mathrm{~S}\right]^{*+}, 336$ $\left[\mathrm{C}_{13} \mathrm{H}_{16} \mathrm{Cl}_{2} \mathrm{NO}_{3} \mathrm{~S}\right]^{+}, \quad 246 \quad\left[\mathrm{C}_{13} \mathrm{H}_{16} \mathrm{~N}_{3} \mathrm{O}_{2}\right]^{+}, \quad 120$ $\left[\mathrm{C}_{7} \mathrm{H}_{6} \mathrm{NO}\right]^{+}, 83\left[\mathrm{C}_{5} \mathrm{H}_{9} \mathrm{~N}\right]^{+}$.

\section{1-(3,5-Dichloro-2-ethoxyphenylsulfonyl)- $N$-(4- dimethylamino)benzylidene) piperidin-4- carbohydrazide (8f)}

Off white powder; Yield: $100 \%$; IR ( $\mathrm{KBr})$ : $\mathrm{U}_{\max }$ : $3128(\mathrm{Ar}-\mathrm{H}), 3000(\mathrm{C}-\mathrm{H}), 1665(\mathrm{C}=\mathrm{N}), 1597$ (Aromatic C=C), $1405\left(-\mathrm{SO}_{2}\right), 1102(\mathrm{C}-\mathrm{O}-\mathrm{C}) ;{ }^{1} \mathrm{H}-$ NMR $\left(400 \mathrm{MHz}, \mathrm{CDCl}_{3}\right): \delta 8.42\left(\mathrm{~s}, 1 \mathrm{H}, \mathrm{H}-7 \mathrm{C}^{\prime \prime}\right)$, $7.93\left(\mathrm{~d}, J=2.8 \mathrm{~Hz}, 1 \mathrm{H}, \mathrm{H}-6^{\prime}\right), 7.89(\mathrm{~d}, J=2.4 \mathrm{~Hz}$, $\left.1 \mathrm{H}, \mathrm{H}-4^{\prime}\right), 7.35$ (d, J = 8.4 Hz, 2H, H-2" \& H-6"), $6.66\left(\mathrm{~d}, J=8.8 \mathrm{~Hz}, 2 \mathrm{H}, \mathrm{H}-3^{\prime \prime} \& \mathrm{H}-5 "\right), 4.25(\mathrm{q}, J=$ $\left.6.8 \mathrm{~Hz}, 2 \mathrm{H}, \mathrm{H}-\mathrm{7}^{\prime}\right), 3.02$ (s, 6H, H-8" \& H-9"), 3.69$3.56\left(\mathrm{~m}, 2 \mathrm{H}, \mathrm{H}_{e}-2 \& \mathrm{H}_{e}-6\right), 3.40-3.20(\mathrm{~m}, 1 \mathrm{H}, \mathrm{H}-$ 4), 2.86-2.80 (m, $2 \mathrm{H}, \mathrm{H}_{\mathrm{a}}-2$ \& $\left.\mathrm{H}_{\mathrm{a}}-6\right), 1.96-1.84$ (m, $4 \mathrm{H}, \mathrm{H}-3$ \& H-5), 1.43 (t, $\left.J=6.4 \mathrm{~Hz}, 3 \mathrm{H}, \mathrm{H}-8^{\prime}\right)$; $\mathrm{El}-$ MS: $m / z 526[\mathrm{M}]^{+}, 379\left[\mathrm{C}_{14} \mathrm{H}_{17} \mathrm{Cl}_{2} \mathrm{~N}_{2} \mathrm{O}_{4} \mathrm{~S}\right]^{++}, 336$
$\left[\mathrm{C}_{13} \mathrm{H}_{16} \mathrm{Cl}_{2} \mathrm{NO}_{3} \mathrm{~S}\right]^{+}, \quad 273\left[\mathrm{C}_{15} \mathrm{H}_{21} \mathrm{~N}_{4} \mathrm{O}\right]^{+}$,

$\left[\mathrm{C}_{9} \mathrm{H}_{11} \mathrm{~N}_{2}\right]^{+}, 83\left[\mathrm{C}_{5} \mathrm{H}_{9} \mathrm{~N}\right]^{+}$.

1-(3,5-Dichloro-2-ethoxyphenylsulfonyl)- $N$ (3,4-dimethoxybenzylidene)piperidine-4carbohydrazide $(8 \mathrm{~g})$

Light grey powder; Yield: $96 \%$; IR ( $\mathrm{KBr})$ : $U_{\max }$ : 3075 (Ar-H), $2881(\mathrm{C}-\mathrm{H}), 1664(\mathrm{C}=\mathrm{N}), 1588$ (Aromatic $\mathrm{C}=\mathrm{C}), 1395\left(-\mathrm{SO}_{2}\right), 1145(\mathrm{C}-\mathrm{O}-\mathrm{C}) ;{ }^{1} \mathrm{H}-$ NMR $\left(400 \mathrm{MHz}, \mathrm{CDCl}_{3}\right): \delta 8.47$ (br s, $1 \mathrm{H}, \mathrm{CONH}$ ), $8.41(\mathrm{~s}, 1 \mathrm{H}, \mathrm{H}-7 "), 7.85(\mathrm{~d}, J=2.8 \mathrm{~Hz}, 1 \mathrm{H}, \mathrm{H}-$ 6'), $7.78\left(\mathrm{~d}, J=2.4 \mathrm{~Hz}, 1 \mathrm{H}, \mathrm{H}-4^{\prime}\right), 7.54(\mathrm{~d}, J=1.6$ $\mathrm{Hz}, 1 \mathrm{H}, \mathrm{H}-2$ "), 7.36 (dd, $J=8.4,1.6 \mathrm{~Hz}, 1 \mathrm{H}, \mathrm{H}-$ 6"), $7.03(\mathrm{~d}, J=8.4 \mathrm{~Hz}, 1 \mathrm{H}, \mathrm{H}-5 "), 4.23$ (q, $J=$ $\left.7.2 \mathrm{~Hz}, 2 \mathrm{H}, \mathrm{H}-7^{\prime}\right), 3.89$ (s, 3H, $\left.\mathrm{OCH}_{3}-8 "\right), 3.87$ (s, $3 \mathrm{H}, \mathrm{OCH}_{3}-9$ "), 3.79-3.56 (m, $2 \mathrm{H}, \mathrm{H}_{e}-2$ \& $\left.\mathrm{H}_{e}-6\right)$, 3.40-3.20 (m, 1H, H-4 ), 2.86-2.80 (m, 2H, $\mathrm{H}_{\mathrm{a}}-2$ \& $\mathrm{H}_{\mathrm{a}}-6$ ), 1.96-1.74 (m, 4H, H-3 \& H-5), 1.43 (t, $J=$ $\left.6.8 \mathrm{~Hz}, 3 \mathrm{H}, \mathrm{H}-8^{\prime}\right)$; EIMS: $\mathrm{m} / \mathrm{z} 543\left[\mathrm{M}^{+}{ }^{+}, 379\right.$ $\left[\mathrm{C}_{14} \mathrm{H}_{17} \mathrm{Cl}_{2} \mathrm{~N}_{2} \mathrm{O}_{4} \mathrm{~S}\right]^{+}, 364\left[\mathrm{C}_{14} \mathrm{H}_{17} \mathrm{Cl}_{2} \mathrm{NO}_{4} \mathrm{~S}\right]^{+}, 336$ $\left[\begin{array}{llll}\left.\mathrm{C}_{13} \mathrm{H}_{16} \mathrm{Cl}_{2} \mathrm{NO}_{3} \mathrm{~S}\right]^{+}, & 290 & {\left[\mathrm{C}_{15} \mathrm{H}_{20} \mathrm{~N}_{3} \mathrm{O}_{3}\right]^{+},} & 126\end{array}\right.$ $\left[\mathrm{C}_{9} \mathrm{H}_{10} \mathrm{~N}_{2} \mathrm{O}\right]^{+}, \quad 164 \quad\left[\mathrm{C}_{9} \mathrm{H}_{10} \mathrm{NO}_{2}\right]^{++}, \quad 147$ $\left[\mathrm{C}_{5} \mathrm{H}_{9} \mathrm{NO}_{2} \mathrm{~S}\right]^{++}, 83\left[\mathrm{C}_{5} \mathrm{H}_{9} \mathrm{~N}\right]^{+}$.

1-(3,5-Dichloro-2-ethoxyphenylsulfonyl)- $N$-(2nitrobenzylidene)piperidine-4-carbohydrazide (8h)

Light green powder; Yield: $96 \%$; IR (KBr): $U_{\max }$ : 3015 (Ar-H), $2910(\mathrm{C}-\mathrm{H}), 1645(\mathrm{C}=\mathrm{N}), 1598$ (Aromatic $\mathrm{C}=\mathrm{C}), 1406\left(-\mathrm{SO}_{2}\right), 1122(\mathrm{C}-\mathrm{O}-\mathrm{C}) ;{ }^{1} \mathrm{H}-$ NMR $\left(400 \mathrm{MHz}, \mathrm{CDCl}_{3}\right): \delta 8.47$ (br s, $1 \mathrm{H}, \mathrm{CONH}$ ), $8.30(\mathrm{~s}, 1 \mathrm{H}, \mathrm{H}-7 "), 8.03(\mathrm{~d}, J=8.0 \mathrm{~Hz}, 1 \mathrm{H}, \mathrm{H}-$ 6"), 7.94 (d, $J=7.8 \mathrm{~Hz}, 1 \mathrm{H}, \mathrm{H}-3$ "), 7.80 (d, $J=$ $\left.2.8 \mathrm{~Hz}, 1 \mathrm{H}, \mathrm{H}-6^{\prime}\right), 7.58$ (d, J = 2.4 Hz, 1H, H-4'), 7.67-7.61 (m, 2H, H-4" \& H-5"), 4.03 (q, $J=6.8$ $\left.\mathrm{Hz}, 2 \mathrm{H}, \mathrm{H}-7^{\prime}\right), 3.69-3.56\left(\mathrm{~m}, \mathrm{H}_{e}-2 \& \mathrm{H}_{e}-6\right)$, 3.40$3.20(\mathrm{~m}, 1 \mathrm{H}, \mathrm{H}-4), 2.86-2.80\left(\mathrm{~m}, 2 \mathrm{H}, \mathrm{H}_{\mathrm{a}}-2\right.$ \& $\mathrm{H}_{\mathrm{a}}-6$ ), 1.96-1.84 (m, 4H, H-3 \& H-5), $1.43(\mathrm{t}, J=6.4$ $\left.\mathrm{Hz}, 3 \mathrm{H}, \mathrm{H}-8^{\prime}\right)$; El-MS: $\mathrm{m} / \mathrm{z} 528$ [M] $^{++}, 379$ $\left[\mathrm{C}_{14} \mathrm{H}_{17} \mathrm{Cl}_{2} \mathrm{~N}_{2} \mathrm{O}_{4} \mathrm{~S}\right]^{+}, 364\left[\mathrm{C}_{14} \mathrm{H}_{17} \mathrm{Cl}_{2} \mathrm{NO}_{4} \mathrm{~S}\right]^{+}, 336$ $\left[\mathrm{C}_{13} \mathrm{H}_{16} \mathrm{Cl}_{2} \mathrm{NO}_{3} \mathrm{~S}\right]^{+}, \quad 275 \quad\left[\mathrm{C}_{13} \mathrm{H}_{15} \mathrm{~N}_{4} \mathrm{O}_{3}\right]^{+}, 149$ $\left[\mathrm{C}_{7} \mathrm{H}_{5} \mathrm{~N}_{2} \mathrm{O}_{2}\right]^{+}, 126\left[\mathrm{C}_{6} \mathrm{H}_{10} \mathrm{~N}_{2} \mathrm{O}\right]^{+}, 83\left[\mathrm{C}_{5} \mathrm{H}_{9} \mathrm{~N}\right]^{+}$.

\section{Biological studies}

The results for in vitro antibacterial activity against Salmonella typhi, Escherichia coli, Pseudomonas aeruginosa, Bacillus subtilis and Staphylococcus aureus are presented in Table 1 and those for in vitro a-glucosidase inhibitory activity are presented in Table 2 .

\section{Molecular docking}

With regard to hydroxyl-containing compounds (8b and $8 \mathrm{e}$ ), the docking conformation of $\mathbf{8 e}$ (ortho analogue) showed good interaction network as well as good docking score compared 


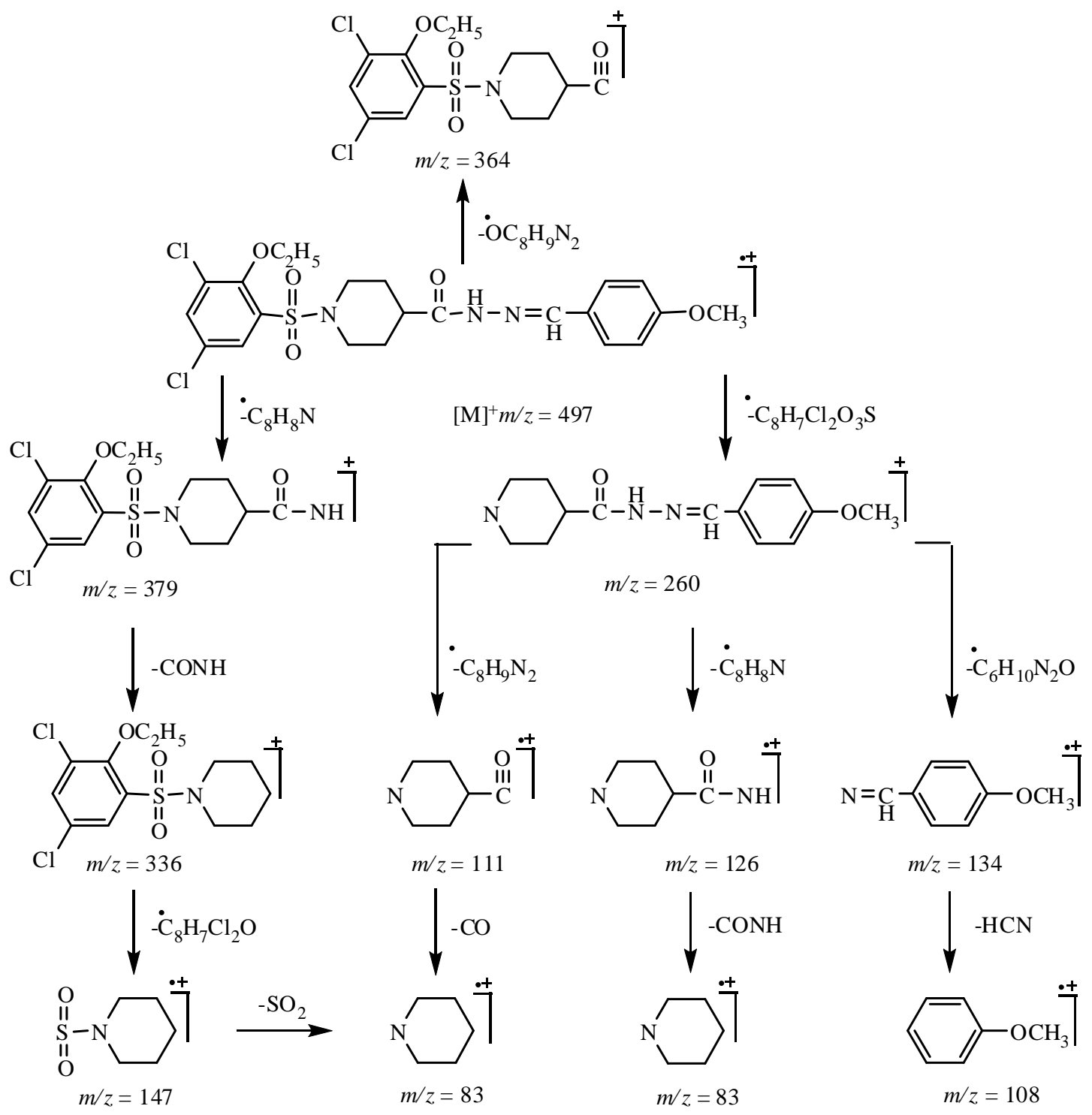

Figure 1: Mass fragmentation pattern of 1-[(3,5-dichloro-2-ethoxyphenyl)sulfonyl]- $N$ '-(4-methoxybenzylidene)piperidin-4-carbohydrazide (8a)

$\left.{ }^{1}\right)$ and $-\mathrm{N}=\mathrm{CH}-\left(1678 \mathrm{~cm}^{-1}\right)$. In the aromatic region of the ${ }^{1} \mathrm{H}$-NMR spectrum, two $m$-coupled signals of the phenylsulfonyl group with one proton integration, a J-value of 2.4 and $2.8 \mathrm{~Hz}$, appeared at $\delta 7.58$ (para proton to sulfonyl group) and $\delta 7.80$ (ortho proton to sulfonyl group). The signals resonating at $\delta 7.71(\mathrm{~d}, J=$ $8.2 \mathrm{~Hz}, 2 \mathrm{H}, \mathrm{H}-\mathrm{2}^{\prime \prime}$ \& $\left.\mathrm{H}-6 "\right)$ and $7.21(\mathrm{~d}, J=8.4 \mathrm{~Hz}$, $2 \mathrm{H}, \mathrm{H}-3$ " \& $\mathrm{H}-5$ ") were assigned to the parasubstituted benzene ring due to the large coupling constant and symmetry of the molecule. In the aliphatic section, the multiplets appearing at $\delta$ 3.89-3.84 (m, 2H, $\left.\mathrm{H}_{e}-2 \& \mathrm{H}_{e}-6\right)$, 3.36-3.20 (m, $1 \mathrm{H}, \mathrm{H}-4), 2.86-2.80\left(\mathrm{~m}, 2 \mathrm{H}, \mathrm{H}_{\mathrm{a}}-2\right.$ \& $\left.\mathrm{H}_{\mathrm{a}}-6\right)$ and 1.96-1.80 (m, 4H, H-3 \& H-5) for nine protons were assigned to the piperidine ring. The two quartet and triplet signals at $\delta 4.23(\mathrm{q}, J=6.4$ $\left.\mathrm{Hz}, 2 \mathrm{H}, \mathrm{H}-7^{\prime}\right)$ and $\delta 1.43\left(\mathrm{t}, J=6.4 \mathrm{~Hz}, 3 \mathrm{H}, \mathrm{H}-8^{\prime}\right)$ were assigned to five protons of the ethoxy group, ortho to sulfonyl group. A chemical shift value at $\delta 3.81$ was assigned to singlet of the methoxy group attached to one of the benzene rings. The singlet appearing at $\delta 8.38(\mathrm{~s}, 1 \mathrm{H}, \mathrm{H}-$ $7 ")$, due to a downfield shift, was attributed to a proton of the imine group. The structure of $\mathbf{8 a}$ was substantiated and designated 1-[(3,5dichloro-2-ethoxyphenyl)sulfonyl]- $N^{\prime}$-(4-methoxybenzylidene)piperidin-4-carbohyrazide. The structure of the other compounds was likewise established.

The screening of all these synthesized compounds against Gram-positive and -negative bacterial strains showed potent antibacterial activity in all but a few. Compounds $\mathbf{8 e}$ and $\mathbf{8 c}$ exhibited good inhibition percentage and MIC values against all bacterial strains, possibly due to the presence of a 2-hydroxyphenyl and 3nitrophenyl group, respectively, in comparison to ciprofloxacin, taken as reference standard. 

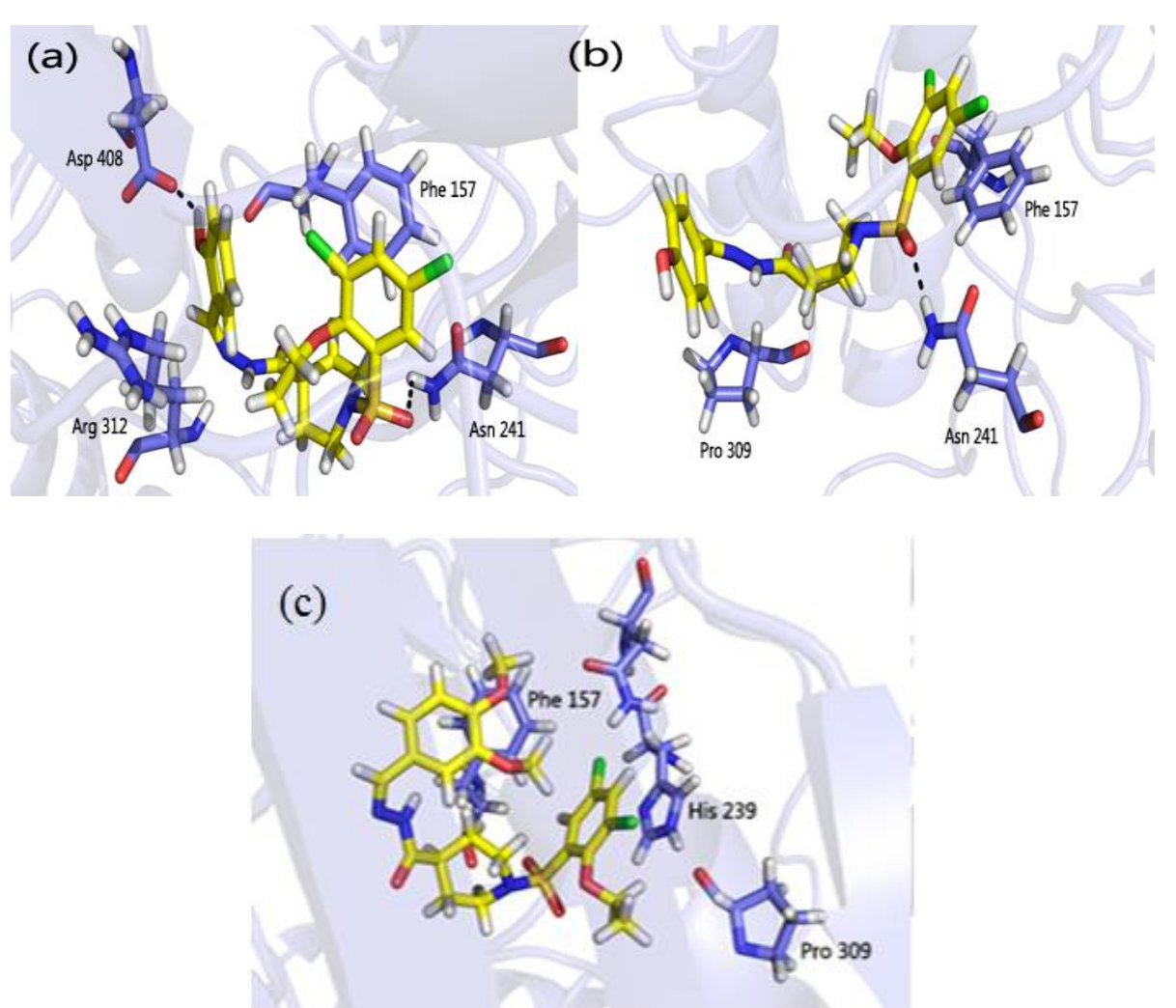

Figure 2: Binding models of compounds; (a): Compound 8e nicely binds to a-glucosidase through Asn 241 and Asp and two $\pi-\pi$ interactions with Phe 157 and Arg 312. (b): Compound 8b binds well to $\alpha$-glucosidase through Asn 241 and one $\pi-\pi$ interaction with Phe 157. (c): Compound $\mathbf{8 g}$ binds to a-glucosidase and shows two $\pi-\pi$ interactions with Phe 157 and His 239

Compounds $8 \mathrm{a}, \mathbf{8 b}, \mathbf{8 d}, \mathbf{8 f}, \mathbf{8 g}$ and $8 \mathrm{~h}$ demonstrated good to moderate activity against both Gram-positive and -negative bacterial strains. S. typhi was best inhibited by molecule 3 (ethyl ester) with a MIC of $7.99 \pm 0.86 \mu \mathrm{M}$ and $8 \mathrm{e}$ (bearing 2-hydroxyphenyl group) with a MIC of $8.00 \pm 0.54 \mu \mathrm{M}$ relative to $7.12 \pm 0.21 \mu \mathrm{M}$ for ciprofloxacin. Against E. coli, molecule 6 (carbohydrazide) with a MIC of $8.05 \pm 0.52 \mu \mathrm{M}$ and $8 f$ (bearing a 4-(dimethylamino)phenyl group) with a MIC of $8.21 \pm 0.83 \mu \mathrm{M}$ were the most effective in comparison to $7.05 \pm 0.28 \mu \mathrm{M}$ for the reference. The synthesized compounds showed relatively moderate activity against $B$. subtilis with the lowest MIC for $8 \mathrm{c}$ (bearing a 3nitrophenyl group) at $8.56 \pm 0.63 \mu \mathrm{M}$ as compared to $7.65 \pm 0.48 \mu \mathrm{M}$ for the standard. The moderate to excellent activity against Staphylococcus aureus rendered 3 (ethyl ester) and $8 \mathrm{c}$ (bearing a 3-nitrophenyl group) the best ones with MIC values of $8.43 \pm 0.79$ and $8.86 \pm$ $0.29 \mu \mathrm{M}$ in comparison to $7.89 \pm 0.27 \mu \mathrm{M}$.

Similarly, evaluation of a-glucosidase inhibitory activity of all synthesized compounds showed moderate activity except $\mathbf{8 a}, \mathbf{8 c}$ and $\mathbf{8 h}$, the inactive ones. Good activity was shown by $8 \mathrm{e}$ and $8 d$ with respective $I_{50}$ values of $40.62 \pm$ 0.07 and $48.64 \pm 0.08 \mu \mathrm{M}$ in comparison to 38.25 $\pm 0.12 \mu \mathrm{M}$ for acarbose, the positive control. The activity of $\mathbf{8 e}$ and $\mathbf{8 d}$ was probably due to the presence of a hydroxyl and chloro group in these compounds. Compounds $\mathbf{8 b}, \mathbf{5}$ and $\mathbf{8 f}$ were less active, and 3,6 and $8 \mathrm{~g}$ had very low poorly active. However, $\mathbf{8 a}$ and $\mathbf{8 b}$ showed outstanding activity and could be further evaluated for the treatment of type-2 diabetes.

\section{CONCLUSION}

The biological activity data obtained demonstrate that the target compounds are significant inhibitors of bacterial growth and a-glucosidase activity. On the basis of the aforementioned results, these newly synthesized compounds may be further developed for the treatment of type-2 diabetes and bacterial infections.

\section{DECLARATIONS}

\section{Acknowledgement}

The authors are thankful to the Higher Education Commission of Pakistan for financial support for this work and for access to facilities for spectroscopic analysis. Dr A Leyva (USA) helped with English editing of the manuscript. 


\section{Conflict of Interest}

No conflict of interest associated with this work.

\section{Contribution of Authors}

The authors declare that this work was done by the authors named in this article and all liabilities pertaining to claims relating to the content of this article will be borne by them.

\section{Open Access}

This is an Open Access article that uses a funding model which does not charge readers or their institutions for access and distributed under the terms of the Creative Commons Attribution License (http://creativecommons.org/licenses/by/ 4.0) and the Budapest Open Access Initiative (http://www.budapestopenaccessinitiative.org/rea d), which permit unrestricted use, distribution, and reproduction in any medium, provided the original work is properly credited.

\section{REFERENCES}

1. Elmali A, Kabak M, Elerman Y. Keto-enol tautomerism, conformations and structure of N-(2-hydroxy-5methylphenyl)-2-hydroxybenzaldehydeimine. $J \mathrm{Mol}$ Struct 2000; 477: 151-158.

2. Chavan AA, Pai NR. Synthesis and biological activity of $\mathrm{N}$-substituted-3-chloro-2-azetidinones. Molecules 2007; 12: 2467-2477.

3. Mittal P, Uma V. Coordination behaviour and biological activity of unsymmetrical bis-hydrazone towards manganese (II) ion. Int J Chem Sci 2008; 6: 1050-1060.

4. Mittal P, Uma V. Stability constants and antimicrobial studies of metal (II) complexes with cinnamaldehyde-(2hydroxybenzylidene) hydrazide. Asian J Chem 2009; 21: 1230-1238.

5. Palet PR, Thaker BT, Zele S. Preparation and characterization of some lanthanide complexes involving a heterocyclic $\beta$ - diketone. Indian J Chem 1999; 38: 563-567.

6. Sharma MC, Kohli DV, Sharma S, Sharma AD. Synthesis and antihypertensive activity of Schiff bases of 4'-(6chloro-5-nitro- 2-[4-(3-substituted-phenyl-acryloylamino)- phenyl]-benzimidazole-1-ylmethyl)-biphenyl-2-carboxylic acids. Adv Appl Sci Res 2010; 1: 120-132.

7. Jungreis E, Thabet S. Analytical Applications of Azomethine derivatives, New York: Marcell Dekker; 1969. $149 \mathrm{p}$.

8. Arulmurugan $S$, Helen $P$, Kavitha $B R$, Venkatraman. Biological activities of schiff base and its complexes. Rasyan J Chem 2010; 3: 385-410.

9. Kaspady M, Narayanaswamy VK, Raju M, Rao GK. Synthesis, antibacterial activity of 2, 4-disubstituted oxazoles and thiazoles as bioesters. Lett Drug Des Discov 2009; 6: 21-28.

10. Bruni $C B$, Sica V, Auricchio F, Covelli I. Further kinetic and structural characterisation of the lysosomal $\alpha-D$ glucoside glucohydrolase from cattle liver. Biochim Biophys Acta 1970; 212: 470-477.

11. Nichols BL, Avery SE, Karnsakul W, Jahoor F, Sen $P$, Swallow DM, Luginbuehl U, Hahn D, Sterchi EE. Congenital maltase-glucoamylase deficiency associated with lactase and sucrase deficiencies. I Pediatr Gastroenterol Nutr 2002; 35: 573-579.

12. De-Laar FAV. Alpha-glucosidase inhibitors in the early treatment of type 2 diabetes. Vasc Health Risk Manag 2008; 4: 1189-1195.

13. De-Laar FAV, Lucassen PL, Akkermans RP, De-Lisdonk EHV, Rutten GE, Weel CV. Diabetes Care 2005; 28 : 154-163.

14. Aziz-ur-Rehman, Nafeesa K, Abbasi MA, Kashifa $H$, Rasool S, Ahmed I, Arshad S. Synthesis, characterization and biological screening of various $S$ substituted derivatives of 5-(3-nitrophenyl)-1,3,5oxadiazole-2-thiol. Pak J Chem 2013; 3: 56-63.

15. Brueggeman GP, Hollingsworth RI. A preparation and screening strategy for glucosidase inhibitors. Tetrahedron 2001; 57: 8773-8778.

16. Allouche AR. Gabedit - A graphical user interface for computational chemistry softwares. J Comput Chem 2011; 32: 174-182.

17. Stewart JP. Optimization of parameters for semiempirical methods V: Modification of NDDO approximations and application to 70 elements. J Mol Model 2007; 13: 11731213.

18. Trott O, Olson AJ. AutoDock Vina: improving the speed and accuracy of docking with a new scoring function, efficient optimization, and multithreading. J Comput Chem 2010; 31: 455-461. 